\title{
Antitumor effects of oncolytic adenovirus armed with Drosophila melanogaster deoxyribonucleoside kinase in colorectal cancer
}

\author{
SHUAI MA ${ }^{1}$, WENZHI QU ${ }^{2}$, LIANG MAO $^{1}$, ZHI ZHU $^{1}$, LANLING JIA ${ }^{1,3}$, LEI ZHAO $^{4}$ and XINYU ZHENG ${ }^{1,3}$ \\ ${ }^{1}$ Department of Surgical Oncology, Department of General Surgery, First Affiliated Hospital, \\ and ${ }^{2}$ Department of Surgical Oncology, Department of General Surgery, Fourth Affiliated Hospital, \\ China Medical University, Shenyang; ${ }^{3}$ Laboratory 1 , Cancer Institute, China Medical University, Shenyang; \\ ${ }^{4}$ Center of Experiment Technology and Medical Research, China Medical University, Shenyang, P.R. China
}

Received November 3, 2011; Accepted December 28, 2011

DOI: $10.3892 /$ or.2012.1665

\begin{abstract}
Drosophila melanogaster multisubstrate deoxyribonucleoside kinase (Dm-dNK) was applied as a cancer gene therapeutic approach. To improve the antitumor effect of Dm-dNK, a novel suicide gene system based on an oncolytic adenovirus vector was developed to produce therapeutic effects towards colorectal cancer cells. We constructed an oncolytic adenoviral vector (ZD55), which was designed by deletion of the E1B-55 kDa gene for selective replication in tumor cells, containing suicide gene (Dm-dNK) driven by a cytomegalovirus promoter. We analysed the expression and activity of Dm-dNK in colorectal cancer cells (HCT-116 and SW620) via reverse transcription (RT)-PCR and enzyme assay. We assessed selective cytotoxic effects of Dm-dNK with the presence of the analogs (E)-5-(2-bromovinyl)-2'-deoxyuridine (BVDU), difluorodeoxycytidine $(\mathrm{dFdC})$ or $1-\beta-\mathrm{D}$-arabinofuranosylthymine (ara-T) by MTT and FACS; the variation of oncolytic adenovirus was detected by titer assay and western blot analysis. Our data showed that ZD55-Dm-dNK mediated high expression of Dm-dNK in HCT-116 and SW620 cancer cell lines and low levels of expression in WI-38 and MRC-5 normal cells, strong cytotoxicity was observed only in tumor cells after ZD55Dm-dNK infection combined with nucleoside analogs (NA). When ZD55-Dm-dNK was combined with BVDU or dFdC, it produced a synergistic inhibitive effect of adenovirus replication while maintaining specific cancer cell killing activity. The results suggest that the novel oncolytic virus ZD55-Dm-dNK, in combination with NA, has potential as an efficient selective
\end{abstract}

Correspondence to: Professor Xinyu Zheng, Department of Surgical Oncology, Department of General Surgery, First Affiliated Hospital of China Medical University, Nanjing Street 155, Shenyang 110001, P.R. China

E-mail: xyzheng@mail.cmu.edu.cn

Key words: colorectal cancer, Drosophila melanogaster multisubstrate deoxyribonucleoside kinase, oncolytic adenovirus, virus therapy, suicide gene antitumoral agent and may produce synergistic effects in safe control of adenovirus, which is a new promising therapeutic for colorectal cancer.

\section{Introduction}

Colorectal cancer ranks third in developed countries, and fifth in China in both morbidity and mortality (1). Although the incidence of CRC was substantially lower in Asia than in the USA in the mid-twentieth century, the incidence in Japan and China has been rapidly increasing $(2,3)$. Around $50 \%$ of newly diagnosed colorectal cancer patients will die of this disease due to liver metastasis, surgical resection or surgery coupled with systemic chemotherapy of liver metastasis is the currently available treatment for these patients, however, only $25 \%$ of liver metastases are amenable to surgery and $60 \%$ of those patients relapse. These findings underscore the need for improved therapeutic approaches to more efficaciously control this aggressive type of cancer.

Gene-directed enzyme prodrug therapy (GDEPT), also known as suicide gene therapy, using the herpes simplex virus thymidine kinase gene (HSVtk)/ganciclovir (GCV) system delivered by replication-defective viral vectors has been studied extensively as the first-generation gene therapy approach for various cancers $(4,5)$. The GDEPT strategy is based on the introduction of a nucleoside kinase gene into the cancer cells and in most cases, such suicide genes encode non-mammalian enzymes that can convert toxicologically and pharmacodynamically inert agents into highly toxic metabolites (6-8). Now, our study has introduced a new suicide gene Dm-dNK which is derivered from Drosophila melanogaster, the only one kinase of Drosophila melanogaster, combines a wide substrate acceptance with the exceptionally high turnover rates. Dm-dNK plays a key role in the chemotherapeutic treatment of cancer and viral diseases as they catalyze the first, and often rate-limiting step of the nucleoside analogs activation which makes it a unique member of the human and viral nucleoside kinase enzyme family (9). We have showed that Dm-dNK is expressed in human osteosarcoma cells and pancreatic adenocarcinoma cells retaining its enzymatic 
activity, and it is located in the cell nuclei due to a nuclear localization signal in its C-terminal region $(10,11)$.

It is critical to deliver therapeutic gene efficiently into target cancer cells for suicide gene therapy strategies (12-14). Replication-defective adenovirus vectors have been used for different applications for gene therapy of cancers. However, because of limitations involving vector delivery and relatively low levels of gene transfer, such vector systems have been proven relatively inefficient for the treatment of large solid tumors (15). One strategy to overcome these limitations is the use of a conditionally replicating adenovirus (CRAd), also called oncolytic adenovirus, which exert intrinsic antitumor activity through selective replication in lysis cancer cells, causing the spread of progeny viruses in neighboring cancer cells, and eventually lysing the infected cancer cells (16-19). For instance, ONYX-015 an E1B-55 kDa deleted mutant adenovirus with tumor specific replication was introduced for oncolytic gene therapy $(20,21)$. Consequently, several approaches are being explored to increase their potency [defined as the viruses ability to replicate, lyse cells, and spread (22), including 'arming' them with therapeutic transgenes (23)].

By using a conditionally replicating adenovirus ZD55 (24), which is constructed similarly to ONYX-15, we produced a recombiant oncolytic adenovirus ZD55-Dm-dNK, a suicide gene-virotherapy, a marriage of Dm-dNK and oncolytic virus therapy, which is expected to create synergistic effects superior to that of each of the single strategies alone.

\section{Materials and methods}

Construction of the recombinant adenovirus. Complete cDNA sequence of Dm-dNK gene was amplified by PCR from plasmid pGEM-dNK by using the upstream primer GT160 5'-CCG GAA TTC ACC ATG GCG GA GGCA-3' and downstream primer GT161 5'-CGC GGA TCC TCA TTA TCT GGC GAC-3'. Synthetic DNA sequence was released with endonucleases EcoRI and BamHI (New England Biolabs, Beverley, MA) and ligated into plasmid pENTR13 (Sinogene, Shanghai, China) which contains the sequence of mouse cytomegalovirus promoter, multiple clone site and SV40 poly A, to generate pENTR13-dNK. A 1311-bp fragment expression cassette of dNK containing mouse cytomegalovirus promoter was inserted into pZD55 to generate pZD55-CMV-Dm-dNK. Plasmid ZD55-CMV-Dm-dNK was individually transfected into HEK293 cells using Lipofectamine 2000 (Life Technologies) together with adenovirus packaging plasmid pBGHlox (deltaE1, E3) (Microbix Biosystems). After homologous recombination, we obtained one set of replicative adenovirus named ZD55-Dm-dNK, in which the viral E1B55-kDa gene was deleted (Fig. 1A). Large-scale amplification of adenoviruses was carried out in HEK293 cells and purified by ultracentrifugation with cesium chloride.

Cell lines and infectivity. The human colorectal carcinoma cell lines SW620, and HCT-116, were purchased from the Shanghai Cell Collection (Shanghai, China), and the HEK293 cell line (human embryonic kidney cells) was obtained from Cancer institution of China Medical University (Shenyang, China). The normal human embryonic lung fibroblast cell lines WI-38 and MRC-5 were purchased from the ATCC
A
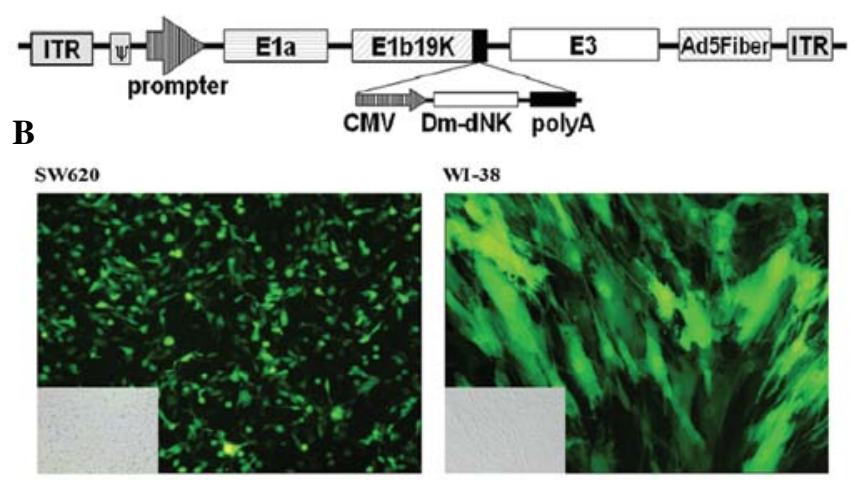

HCT-116

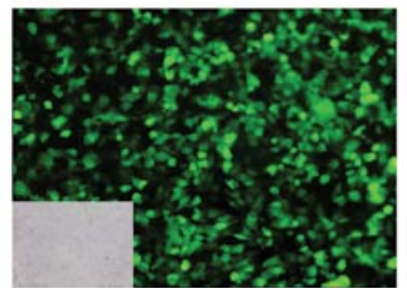

MRC-5

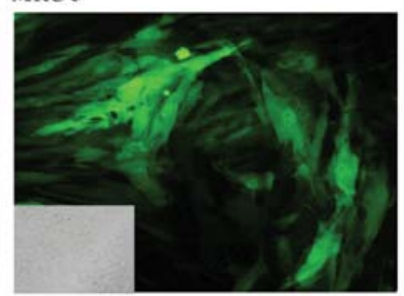

Figure 1. Construction and infectivity of recombinant adenovirus ZD55Dm-dNK. (A) Characterization of ZD55-Dm-dNK, the E1B55-kDa gene (2269-3327bp) was replaced by the expression cassette of Dm-dNK containing cytomegalovirus (CMV) promoter, Dm-dNK and SV40 poly A to generate ZD55-Dm-dNK. ITR, inverted terminal repeat; $\psi$, the adenovirus 5 packing signal. (B) Overview of the infectivity of Type 5 adenovirus in the colorectal carcinoma cells SW620 and HCT-116, the normal human fibroblast cell lines WI-38 and MRC-5. Nearly the same viral infectivity in the four cell lines according to the cells expressed green fluorescence protein.

(American Type Culture Collection). The human colorectal carcinoma cell lines SW620, and HCT-116, were cultured in Leibovitz-15 (Gibco-BRL, Grand Island, NY, USA) and McCOY's 5A (Gibco-BRL) medium, respectively. The HEK293 cell line (human embryonic kidney cells) and the normal human embryonic lung fibroblast cell line WI-38 and the MRC-5 were cultured in Dulbecco's modified Eagle's medium (Gibco-BRL). All the cell lines was cultured with $10 \%$ heat-inactivated fetal bovine serum (Gibco-BRL), $4 \mathrm{mM}$ glutamine, $50 \mathrm{U} / \mathrm{ml}$ penicillin, and $50 \mu \mathrm{g} / \mathrm{ml}$ streptomycin at $37^{\circ} \mathrm{C}$ in a $5 \% \mathrm{CO}_{2}$ humidified incubator.

Comparison of infectivity of Type 5 adenovirus in the cell lines SW620, HCT-116 to WI-38, MRC-5. Cells were examined by using the wild-type 5 adenovirus Ad-GFP (Green fluorescent protein) at multiplicity of infection (MOI) of 1 . The percentage of positive cells was determined under a fluorescence microscope (Nikon, Tokyo, Japan).

$R T$-PCR. ZD55-Dm-dNK and ZD55 were added to the human colorectal carcinoma cells and the normal human embryonic lung fibroblast cells and incubated in proper medium with $10 \%$ FBS, after a medium change, the cells were cultured for 3 more days. The cells were collected and the total RNA was extracted with TRIzol Reagent (Invitrogen, Carlsbad, CA). Briefly, according to the manufaturer's instructions, RT-PCR analysis was performed with $1 \mu \mathrm{g}$ total RNA and oligo (dt)adaptor primer using the two-step RT-PCR (Takara Bio Inc., Shiga, Japan). A cDNA equivalent of $1 \mu \mathrm{g}$ RNA was amplified by PCR using primers specific for the target genes. The 
thermal cycles were: $94^{\circ} \mathrm{C}$ for $0.5 \mathrm{~min}, 60^{\circ} \mathrm{C}$ for $0.5 \mathrm{~min}, 72^{\circ} \mathrm{C}$ for $1 \mathrm{~min}$ for 25 cycles for Dm-dNK ( $779 \mathrm{bp}) ; 94^{\circ} \mathrm{C}$ for $0.5 \mathrm{~min}$, $55^{\circ} \mathrm{C}$ for $0.5 \mathrm{~min}, 72^{\circ} \mathrm{C}$ for $1 \mathrm{~min}$ for 25 cycles for GAPDH (452 bp). The Nucleotide sequences of Dm-dNK primers were as follows: sense 5'-CCG GAA TTC ACC ATG GCG GAG GCA -3'; anti-sense 5'-CGC GGA TCC TCA TTA TCT GGC GAC -3'. The Nucleotide sequences of GAPDH primers were as follows: sense 5'-ACC ACA GTC CAT GCC ATC AC-3'; anti-sense 5'-TCC ACC ACC CTG TTG CTG TA-3'. The amplification products were separated by $2 \%$ agarose gel electrophoresis and visualized by SYBR-Green staining.

Enzyme assay. Cells were cultured in 6-well plates, and crude cell protein extracts were prepared at 5 days after infection at MOI 1. Cell protein extracts were prepared as described (25). The assays were performed in $50 \mathrm{mM}$ Tris- $\mathrm{HCl}, \mathrm{pH}$ 7.6, $5 \mathrm{mM} \mathrm{MgCl} 2,5 \mathrm{mM}$ ATP, $2 \mathrm{mM}$ dithiothreitol, $15 \mathrm{mM} \mathrm{NaF}$, $100 \mathrm{mM} \mathrm{KCl}, 0.5 \mathrm{mg} / \mathrm{ml}$ bovine serum albumin, and $0.6 \mathrm{mg}$ of protein extract in a total volume of $35 \mathrm{ml}$. [methyl $-{ }^{3} \mathrm{H}$ ]dThd $(2.5 \mathrm{mM})$ (Moravek Biochem), were used in the assays and mixed with equivalent amounts of unlabeled substrates. Aliquots of the reaction mixture were spotted on Whatman DE-81 filters after 10,20 and 30 min of incubation at $37^{\circ} \mathrm{C}$. The filters were washed three times in $5 \mathrm{mM}$ ammonium formate. The nucleoside monophosphates were eluted from the filter with $0.5 \mathrm{M} \mathrm{KCL}$, and the radioactivity was determined by scintillation counting.

Cell viability and cytopathic effect assay. Cell viability was measured by methyl thiazolyl tetrazolium (MTT) assay. The human colorectal carcinoma cells and the normal human embryonic lung fibroblast cells was planted at a density of $1 \times 10^{4}$ and $3 \times 10^{3}$ per well in 96-well plates. After incubation for $24 \mathrm{~h}$, the cells were infected with ZD55-Dm-dNK, ZD55, d11520 and wild-type adenovirus (WtAd) at a gradient of MOI from 0 to 10, mock infected acted as control. The MTT assay was performed 5 days post-infection. The absorbance was determined at $570 \mathrm{~nm}$ on a scanning multi-well pectrophotometer with a reference wavelength at $655 \mathrm{~nm}$ (Bio-Rad Laboratories, Hercules, CA). For the combination therapy, cells were infected with ZD55-Dm-dNK or ZD55 at MOI 1 after planting, the medium was replaced for fresh medium $4 \mathrm{~h}$ after infection, after incubation for $24 \mathrm{~h}$, the cells were treated with investigated nucleoside analog (NA) compounds, whose concentration was from 0.01 to $100 \mu \mathrm{M}$. The medium containing the NA was changed daily for 4 days, the survival rates of the cells were performed as described above. Each assay was performed in triplicate. The cells were observed every other day for cytopathic effect (CPE) for 5 days, cells treated with PBS were used as negative control. All microscopy photographs were originally at a magnification of $\mathrm{x} 10$.

Apoptosis assay. The cells were seeded in 6-well plates at a proper density per well and infected with ZD55-Dm-dNK or ZD55 at MOI 1, ZD55-Dm-dNK or ZD55 infection in normal cell lines was the control. The cells were co-cultured for $4 \mathrm{~h}$ and then washed to remove free viral particles. NA was added at a concentration of $1 \mu \mathrm{M} 24 \mathrm{~h}$ after infection. Apoptotic cells were detected using FITC conjugated Annexin-V and propidium iodide (PI) 5 days post-infection. Cells were washed twice with PBS and resuspended in Annexin- $\mathrm{V}$ binging buffer (10 mM HEPES, $140 \mathrm{mM} \mathrm{NaCl}, 5 \mathrm{mM} \mathrm{CaCl}$ ), at a concentration of $1 \times 10^{6} \mathrm{cells} / \mathrm{ml}$. Cell solution $(200 \mu \mathrm{l})$ was added to a $1 \mathrm{ml}$ Eppendorf tube, and $5 \mu \mathrm{l}$ Annexin-V-FITC, and $10 \mu \mathrm{l}$ of PI were added. The tube was gently vortexed and incubated for $15 \mathrm{~min}$ at room temperature in the dark. Binding buffer was added to each tube and the cells were analyzed by cytometry (Becton-Dickinson, Mountain View, CA, USA).

Titer assay. Selective replication of adenovirus was evaluated in colorectal cancer cell lines SW620, HCT-116 and normal cell lines WI-38, MRC-5. Wild-type adenovirus (WtAd) was used as positive control. The colorectal carcinoma cell lines SW620 (5x 10 $/$ well), HCT-116 (5x10 $/$ well), and the normal embryonic

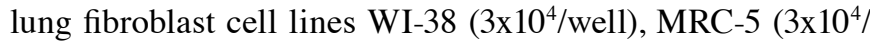
well) were seeded in 24-well plates. After $24 \mathrm{~h}$, the cells were infected with ZD55-Dm-dNK, ZD55, dl1520 and WtAd. Of the above viruses $10^{4} \mathrm{pfu} / \mathrm{ml}$ was added in the culture, the medium was replaced with fresh medium $4 \mathrm{~h}$ after infection. The cells were harvested at $0,1,3$ or 5 days post-infection. The harvested cells were subjected to 3 freeze-thaw cycles to release viral particles. Limiting dilution (TCID50) method in HEK-293 cells was performed to determine the viral titers in cell lysate. Each assay was repeated two to three times, averaged, and expressed as pfu/cell \pm SD.

Western blot analysis of E1A protein. SW620, HCT-116 and WI-38, MRC-5 cells were infected with ZD55-Dm-dNK, ZD55, d11520 and Adwt at MOI of 1. Cells were harvested at 5 days after infection and lysed in a RIPA lysis buffer $(50 \mathrm{mM}$ Tris- $\mathrm{HCl}, 150 \mathrm{mM} \mathrm{NaCl}, 1 \% \mathrm{NP}-40,0.5 \%$ sodium deoxycholate, $0.1 \%$ SDS) with a protease inhibitor cocktail containing phenylmethyl sulfonylfluoride (PMSF) for $30 \mathrm{~min}$. Cell lysates were centrifuged, and protein concentration was determined by BCA protein assay (Kaiji, China). Equal amounts $(80 \mu \mathrm{g})$ of cellular protein were electrophoresed in SDS-polyacrylamide gels purified rabbit anti-adenovirus type $5 \mathrm{E} 1 \mathrm{~A}$ monoclonal antibody (SC-430; Santa Cruz Biotechnology) was diluted to 1:2000 and goat anti-actin at 1:1000 (SC-1615). Binding of the primary antibody was detected by mouse anti-rabbit immunoglobulin (Ig) conjugated to horseradish peroxidase (Amersham, Arlington Heights, IL). ECL reagents were used to detect the signals, according to the manufacturer's instructions (Amersham).

Statistical analysis. Data were analyzed using GraphPad Prism version 5.0 software (GraphPad Software, Inc., San Diego, CA). Each result is expressed as the mean value \pm standard deviation of the mean. Statistical analysis was performed with the Student's t-test to evaluate differences among the experimental groups. Differences were considered significant when the P-value was $<0.05$. The CPE induced by replicationcompetent adenovirus vectors ZD55-Dm-dNK, ZD55, d11520 and Adwt in the human cell lines were compared and analyzed by repeated-measures ANOVA.

\section{Results}

Selective replication of $Z D 55$ adenovirus. The amount of viral particles in both HCT-116 cells and SW620 cells increased 

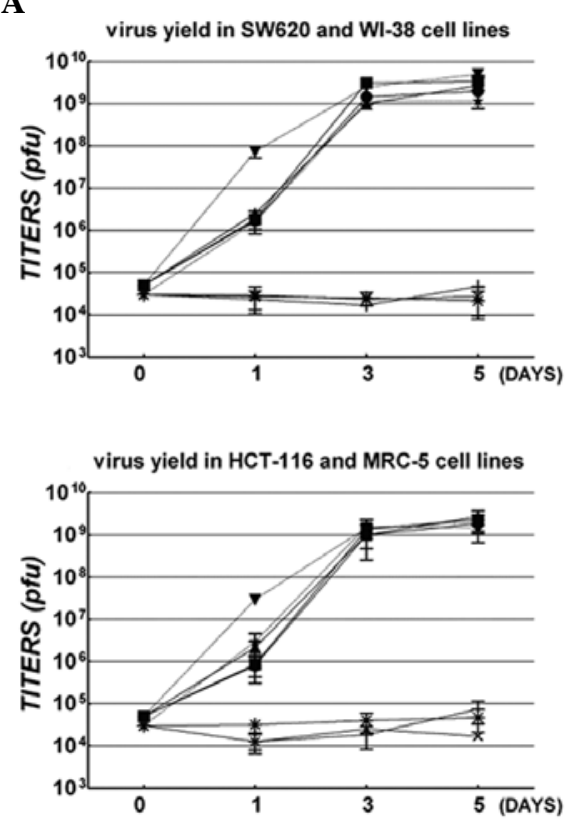

$\mathbf{B}$
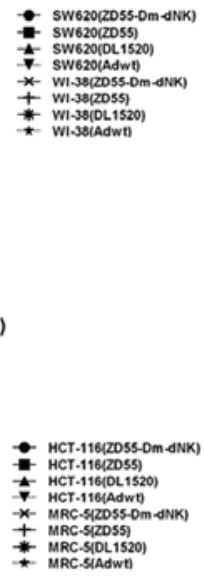

E1A actin

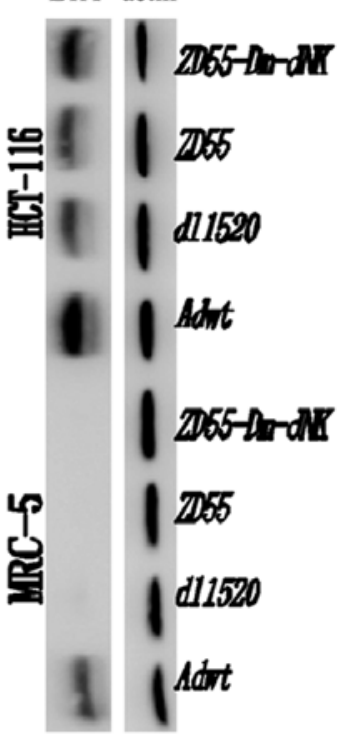

C
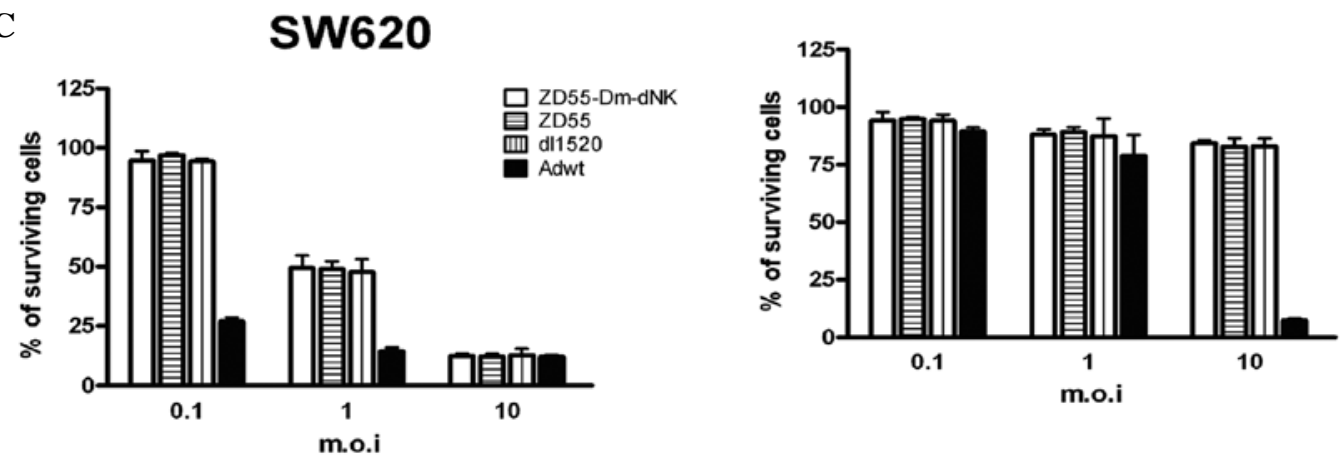

WI-38

Figure 2. Selective replication of ZD55 adenovirus. (A) The amount of viral particles in both HCT-116 cells and SW620 cancer cells increased over time but not in normal cells WI-38 and MRC-5 after infected with ZD55-Dm-dNK, ZD55 and d11520 except for Adwt. (B) Expression of E1A by western blot analysis proved that ZD55-Dm-dNK, ZD55 and d11520 could selectively replicate in tumor cell lines. (C) The survival rate of cancer cells infected with ZD55-Dm-dNK, ZD55 and d11520 was from 10-15\% at MOI 10, whereas, in normal cells, the cytotoxicity of the ZD55-Dm-dNK and ZD55 were not markedly different compared with the d11520 at MOI 10, there was an approximately 80-90\% cell death rate found when we used Adwt.

over time after infection with ZD55-Dm-dNK, ZD55 and d11520, as shown in Fig. 2A. In contrast, the amount of viral particles increased in colorectal cells but not in normal cells WI-38 and MRC-5 except for Adwt 5 days post-infection. Viral titer increased by more than 19952- and 34145-fold in SW620, 18478- and 23263-fold in HCT-116 cells infected with ZD55-Dm-dNK and ZD55, respectively, which was at least 2531-fold higher than in normal cells. Virus production by the ZD55 vectors and d11520 was similar to that by ZD55Dm-dNK in cancer cell lines, but less than that of Adwt. Expression of E1A by western blotting further supported these results (Fig. 2B). The oncolytic effect of the four kinds of adenoviruses was determined by comparing the cytotoxic effect between cancer and normal cells. Cancer cells infected with ZD55-Dm-dNK, ZD55 and d11520 shows that the survival rate of cancer cells was nearly $90 \%$ at $0.1 \mathrm{MOI}$ but was $10-15 \%$ at MOI 10, 5 days after infection. The cancer cells infected with Adwt showed higher cytotoxicity (75-70\%) compared with other viruses (about 10\%) on Day 5 at 0.1 MOI. In normal cells, the cytotoxicity of the ZD55-Dm-dNK and ZD55 were not markedly different compared with the d11520 which is $<20 \%$ cell killing on Day 5 even at MOI 10, although there was an approximately $80-90 \%$ cell death rate found when we used Adwt as shown in (Fig. 2C). These data suggest that there is no difference among ZD55-Dm-dNK, ZD55 and d11520 on its selective replication and oncolytic effect either in tumor or normal cell lines.

Selective expression of Dm- $d N K$. ZD55-Dm-dNK expressed Dm-dNK gene with a much higher efficiency in cancer cells SW620 and HCT-116 compared to the normal cells WI-38 and MRC-5, RT-PCR studies show the selective expression of ZD55-dNK at MOI 1 after 3-day infection (Fig. 3A).

We determined the dThd kinase activity in crude cell extracts of cells incubated with the ZD55-Dm-dNK to verify that the enzymatical activity of Dm-dNK retained and 


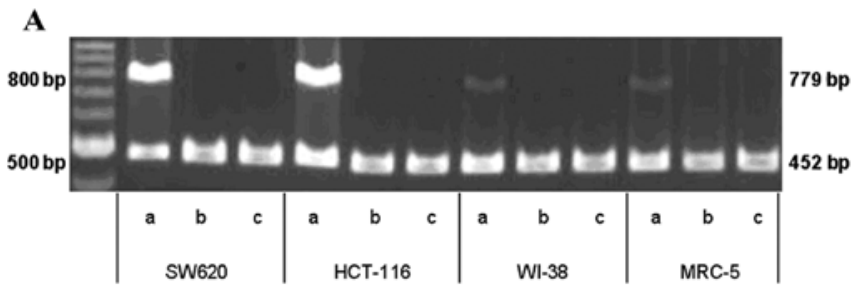

B

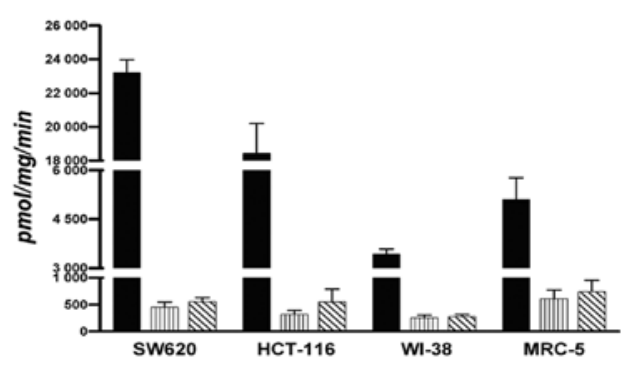

Figure 3. Selective expression of ZD55-Dm-dNK. (A) Colorectal cancer cells SW620, HCT-116 and normal cells WI-38, MRC-5 were infected with (a) ZD55-Dm-dNK, (b) ZD55 or (c) MOCK. RT-PCR analysis indicated the selective expression of ZD55-Dm-dNK, the 779 bp and 452 bp indicate the sizes of the amplified Dm-dNK and GAPDH. (B) Enzymatic activity of Dm-dNK increased exponentially in the cancer cell lines compared with the normal cells. Cells treated with either ZD55 alone and wild-type cells were used as controls.

increased exponentially and selectively in the four cell lines. The kinase activity in HCT-116 cell protein extracts was increased 33- and 58-fold compared with wild-type cells or ZD55 vector infected alone, to that of SW620 cells. However, the normal cells W-I38 and MRC-5 treated with ZD55Dm-dNK were slightly increased 12- to 13 -fold and 7- to 8 -fold, respectively, compared with the uninfected parent cell lines or the cells infected with ZD55 vector alone (Fig. 3B), these experiments showed that human colorectal carcinoma cells transduced with the ZD55-Dm-dNK expressed selective enzymatic activity.

Sensitivity of nucleoside analogs to ZD55-Dm-dNK. The $\mathrm{IC}_{50}$ of uninfected SW620 and HCT-116 cancer cells was 48 and $203 \mu \mathrm{M}$ for BVDU, 68 and $101 \mu \mathrm{M}$ for $\mathrm{dFdC}, 47$ and $163 \mu \mathrm{M}$ for araT. However, the $\mathrm{IC}_{50}$ of ZD55-Dm-dNK infected SW620 and HCT-116 cells was 0.000331 and $0.018125 \mu \mathrm{M}$ for BVDU, 0.000815 and $0.013884 \mu \mathrm{M}$ for $\mathrm{dFdC}, 0.001403$ and $0.026276 \mu \mathrm{M}$ for araT. The highest sensitivity for the colorectal carcinoma cells was detected for BVDU, with a 145015- and 11200-fold decrease in SW620 and HCT-116 compared with the uninfected cells (Fig. 4A).

Similar data were observed by microscopy.ZD55-Dm-dNK infection in combination with BVDU caused the strongest $\mathrm{CPE}$ in colorectal cancer cells, which are more cytotoxic to colorectal cancer cells than araT and $\mathrm{dFdC}$, normal cells MRC-5 and WI-38 are resistant to the infection (Fig. 4B).

Apoptosis of combination effect of ZD55-Dm-dNK or ZD55 plus NA was evaluated by Annexin-V/PI assay. We verified that ZD55-Dm-dNK plus NA could induce cell apoptosis, the combination effect of ZD55-Dm-dNK and NA was significantly greater than that of ZD55 plus NA or ZD55-Dm-dNK alone (Fig. 4C).
Nucleoside analogs activated by Dm-dNK inhibit viral replication. Surprisingly, SW620 cells supported higher levels of replication of the virus when used alone, but in the presence of $1 \mu \mathrm{M} \mathrm{dFdC}$ or BVDU, replication was detected at low levels of $<9$ pfu/cells and $<3$ pfu/cells after ZD55-Dm-dNK infected. Similar scenario was seen in ZD55-Dm-dNK infected HCT-116 cells, viral replication was detected at very low levels of in the presence of $1 \mu \mathrm{M} \mathrm{dFdC}(<19 \mathrm{pfu} /$ cell $)$ or BVDU ( $<4 \mathrm{pfu} / \mathrm{cell})$. Despite a clear increase in replication over time, low doses of BVDU $(1 \mu \mathrm{M})$ or dFdC $(1 \mu \mathrm{M})$ can lead to inhibition of the virus replication after ZD55-Dm-dNK infection in the two colon cancer cell lines. Attenuation was also observed to some extents in normal cell lines (Fig. 5A).

The E1A protein levels in western blot analysis verified that the proper NA activated by Dm-dNK could inhibit the replication of the adenovirus. The E1A proteins in both SW620 and WI-38 cells treated by ZD55-Dm-dNK with BVDU were at moderate, lower levels compared with ZD55 plus dFdC or BVDU or ZD55-Dm-dNK used alone (Fig. 5B), no clear lane of E1A was detected in WI-38 cells.

\section{Discussion}

Cancer cells are genetically and phenotypically complex and frequently harbor multiple abnormalities (26). It is reasonable that single agent such as oncolytic adenovirus one is not sufficient for complete eradication of malignancies. Arming oncolytic adenovirus with suicide genes has become a focus issue in cancer treatment (27). Suicide genes are transferred into cancer cells and express exponentially in the tumor region, can improve the anti-tumor effect of CRAd to some extent. Among various suicide genes, Dm-dNK is a unique member of suicide gene family, which combines a wide substrate aceptance with the exceptionally high turnover rates. In this study, we applied oncolytic adenovirus vectors ZD55 combined with suicide genes Dm-dNK to human colorectal cells for the first time. These data indicated that our newly constructed oncolytic adenoviral vector ZD55-Dm-dNK did not only maintain their intrinsic capacity of viral replication in tumor cells but also induced high and sustained expression of suicide gene to activate the NA for antitumor and antiviral effects. Furthermore, the sensitization of the treatment was specific for cancer cells and did not occur in normal fibroblast cells. The very important issue is that nucleoside analogs phosphorylated by Dm-dNK was able to induce cell death by apoptosis. The results of FACs analysis of our study showed ZD55-Dm-dNK produced more apoptosis of cancer cells than ZD55 vector combined with NA, without causing significant toxic effects to the normal cells. The FACS data are similar to the cell proliferation assays and CPE observations.

Two of the most promising candidate nucleoside analogs to use in conjunction with Dm-dNK gene transfer are the pyrimidine analogs $\mathrm{dFdC}$ and BVDU. BVDU, the first candidate nucleoside analog, was initially developed as an anti-herpetic agent, and the compound has been used to treat patients with varicella zoster infections (28). BVDU shows low toxicity to uninfected cells, but the cells expressing Dm-dNK are highly sensitive to the compound. $\mathrm{dFdC}$ is currently used in the treatment of several types of solid tumors such as pancreatic adenocarcinoma and non-small cell lung cancer (29). 
A

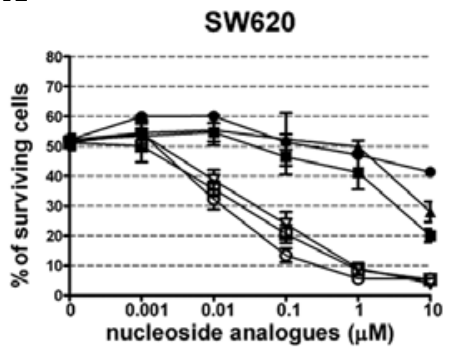

HCT-116

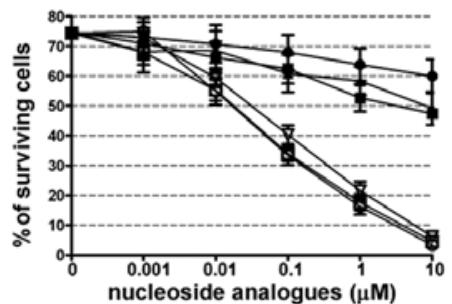

- ZD55-Dm-dNK plus BVDU - ZD55 plus BVD

7. ZD55-Dm-dNK plus dFdc $*$ ZD55 plus dFdc
WI-38

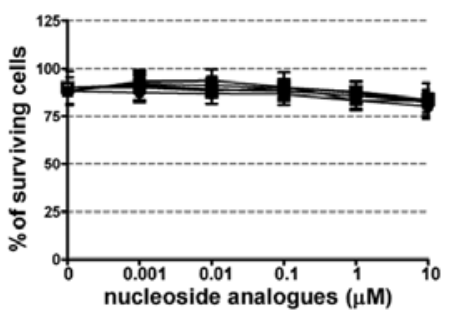

MRC-5

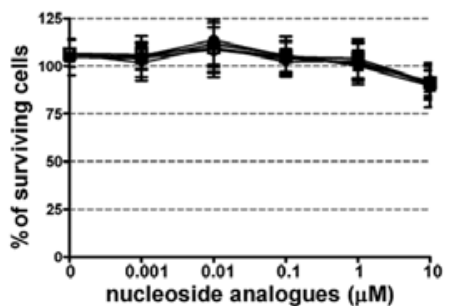

\section{B}
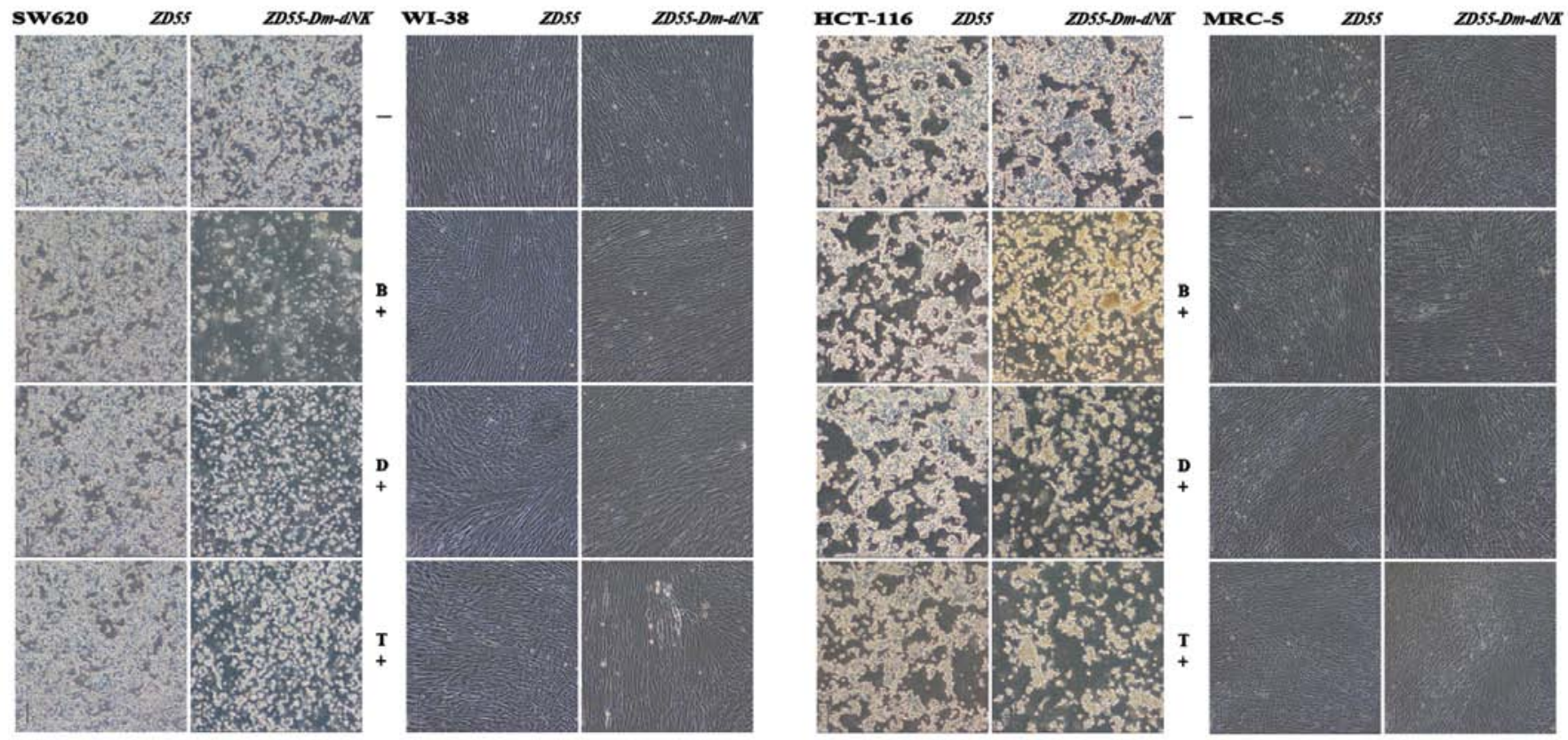

Figure 4. Sensitivity of nucleoside analogs to ZD55-Dm-dNK. (A) Cytotoxicity of ZD55-Dm-dNK or ZD55 was assessed by MTT assay. Treatment of cells with ZD55-Dm-dNK resulted in a dose-dependent reduction of cell viability within 5 days, without affecting normal cells. (B) Cytopathic effect (CPE) of ZD55-Dm-dNK with NA was observed by microscopy in the four cell lines. The (B+) (D+) (T+) (-) indicate ZD55-Dm-dNK or ZD55 plus BVDU, dFdC, araT, or PBS at a concentration of $1 \mu \mathrm{M}$.

Interestingly, the potent increase in cell death in response to combination treatment was not parallel to the viral replication in our study. ZD55-Dm-dNK combined with $\mathrm{dFdC}$ and BVDU killed almost $90 \%$ colon cancer cells while a greatly attenuated replication of ZD55-Dm-dNK was observed. The never previously reported finding may be because the triphosphate form of NA phosphorylated by Dm-dNK induces cell apoptosis while also inducing the death of the adenovirus. As an antiviral agent, $\mathrm{dFdC}$ combined with adenovirus mutant without suicide gene showed a nearly complete inhibition of the adenovirus mutant replication to an undetectable level
(30). Similarly, a much greater attenuation of titers with ZD55-Dm-dNK in colon cancer cells combined with $1 \mu \mathrm{mol} / 1$ $\mathrm{dFdC}$ or BVDU was observed than reported previously, and the cell death in response to combination treatment was not depend on viral replication in our study. The difference may due to the proper antiviral substrate activated by Dm-dNK and different construction between the ZD55 and other adenovirus mutants. The results of western blot analysis and titer assays suggested that the effects of replicated adenovirus could be controlled to some extent by proper type of NA activated by $\mathrm{Dm}-\mathrm{dNK}$ to reduce the side effect. 
C
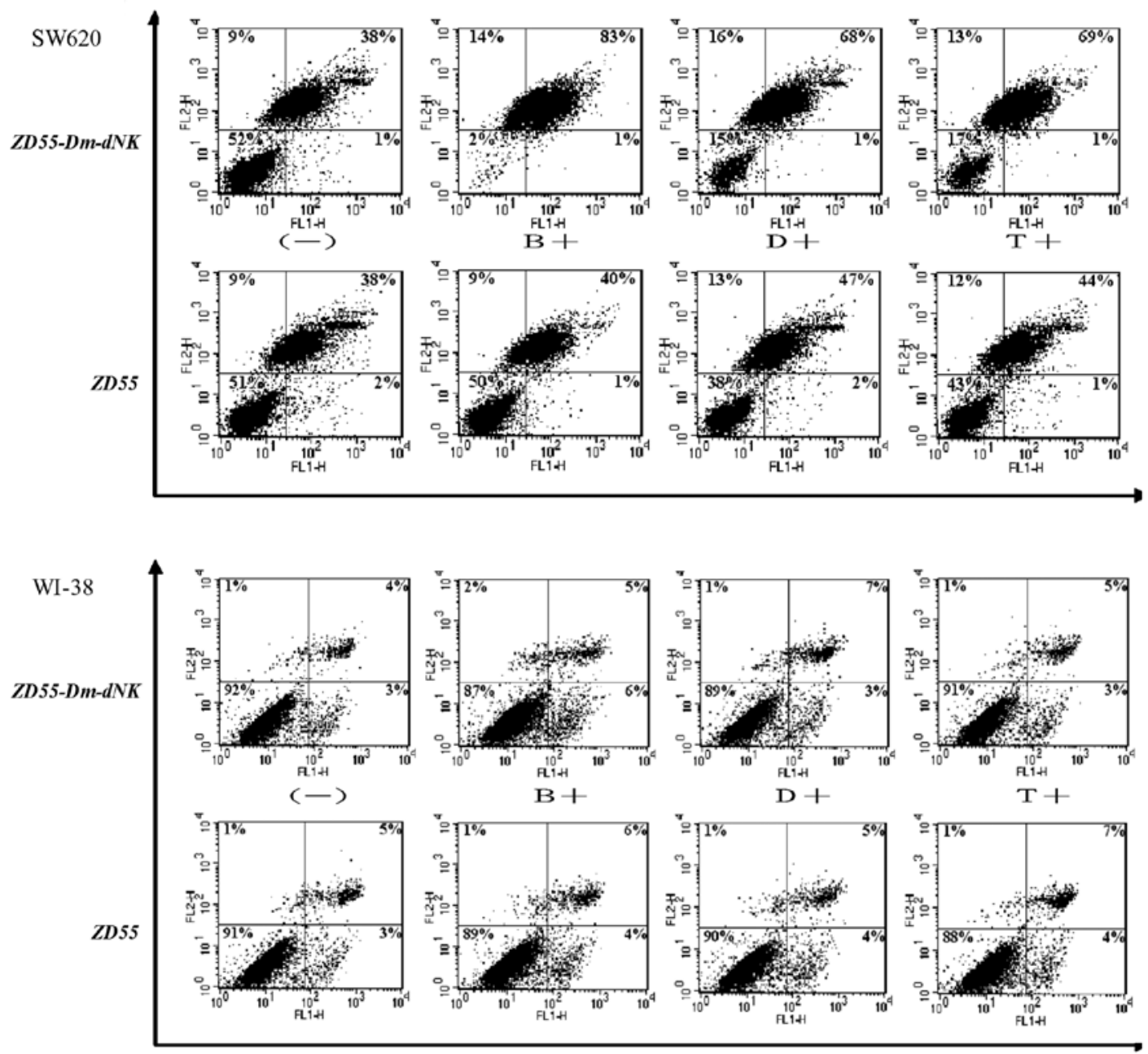

Figure 4. Continued. (C) The Annexin-V-PI assay was used to evaluate combination effect of ZD55-Dm-dNK or ZD55 plus NA. Apoptosis was significantly increased by ZD55-Dm-dNK at MOI 1 in combination with NA at the concentration of $1 \mu \mathrm{M}$. The (B+) (D+) (T+) (-) indicate ZD55-Dm-dNK or ZD55 plus BVDU, dFdC, araT, or PBS at a concentration of $1 \mu \mathrm{M}$.
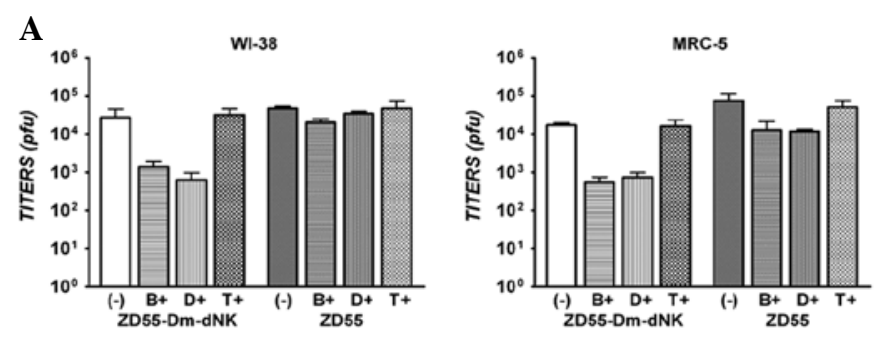

$\mathbf{B}$
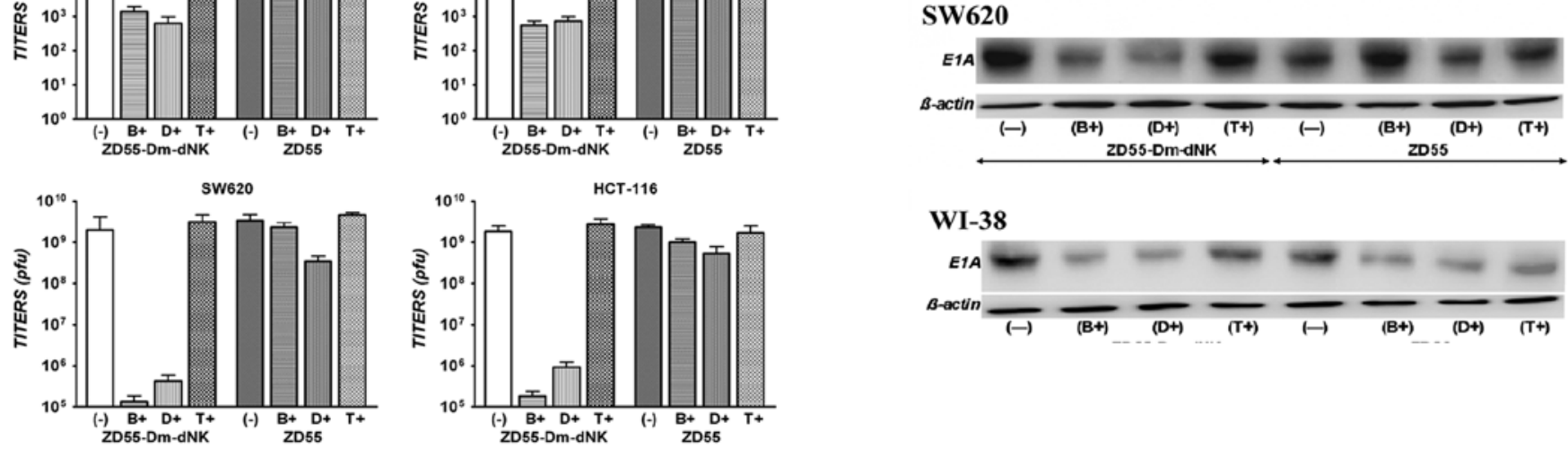

WI-38

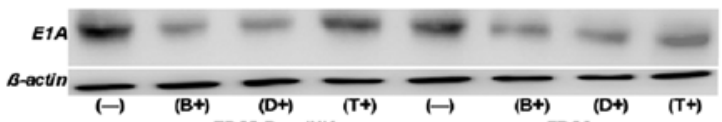

Figure 5. Nucleoside analogs activated by Dm-dNK inhibit viral replication. (A) TCID50 titer assay was performed in the cells infected with ZD55-Dm-dNK or ZD55. The titers of virus ZD55-Dm-dNK with BVDU or dFdC were much lower (up to 1,000-fold) than the level of ZD55 alone or with drugs in SW620, HCT-116 cells, slightly lower (up to 10-fold) in WI-38 and MRC-5 cells. AraT showed less sensitivity than BVDU or dFdC as a substrate to Dm-dNK. (B) Western blot assay of E1A gene expression by ZD55-Dm-dNK, ZD55 in the presence of dFdC or BVDU. With the therapy of ZD55-Dm-dNK combined with BVDU, the E1A of adenovirus was clearly attenuated in SW620 cells. However, in WI-38 cells, ZD55-Dm-dNK and ZD55 did not produce clearly detectable E1A proteins, especially undetectable in the presence of dFdC or BVDU. The Dm-dNK activated dFdC or BVDU prevents viral replication. The (B+) (D+) (T+) (-) indicate ZD55-Dm-dNK or ZD55 plus BVDU, dFdC, araT, or PBS at a concentration of $1 \mu \mathrm{M}$. 
Therefore, this novel ZD55-Dm-dNK might effectively combat colon cancer by decreasing the NA drug concentration at the primary tumor site and provide a potentially safe tool for the adenovirus.

\section{Acknowledgements}

This study was supported by grants from the National Natural Science Foundation of China (no. 81071900 and 81172199); Scientific Research Foundation for Returned Scholars, Ministry of Education of China (2008) and Hi-Tech Research Development Program of China (863 Program, 2006AA02Z493).

\section{References}

1. Winawer SJ: Screening of colorectal cancer: progress and problems. Recent Results Cancer Res 166: 231-244, 2005.

2. Lu JB, Sun XB, Dai DX, et al: Epidemiology of gastroenterologic cancer in Henan Province, China. World J Gastroenterol 9 : 2400-2403, 2003.

3. Yiu HY, Whittemore AS and Shibata A: Increasing colorectal cancer incidence rates in Japan. Int J Cancer 109: 777-781, 2004

4. Moolten FL: Tumor chemosensitivity conferred by inserted herpes thymidine kinase genes: paradigm for a prospective cancer control strategy. Cancer Res 46: 5276-5281, 1986.

5. Osaki T, Tanio Y, Tachibana I, et al: Gene therapy for carcinoembryonic antigen-producing human lung cancer cells by cel type-specific expression of herpes simplex virus thymidine kinase gene. Cancer Res 54: 5258-5261, 1994.

6. Moolten FL: Drug sensitivity (suicide) genes for selective cancer chemotherapy. Cancer Gene Ther 1: 279-287, 1994.

7. Lengler J, Omann M, Duvier D, et al: Cytochrome $\mathrm{P} 450$ reductase dependent inhibition of cytochrome P450 2B1 activity: implications for gene directed enzyme prodrug therapy. Biochem Pharmacol 72: 893-901, 2006.

8. Cai X, Zhou J, Chang Y, Sun X, Li P and Lin J: Targeting gene therapy for hepatocarcinoma cells with the E. coli purine nucleoside phosphorylase suicide gene system directed by a chimeric alpha-fetoprotein promoter. Cancer Lett 264: 71-82, 2008.

9. Zheng X, Johansson M and Karlsson A: Bystander effects of cancer cell lines transduced with the multisubstrate deoxyribonucleoside kinase of Drosophila melanogaster and synergistic enhancement by hydroxyurea. Mol Pharmacol 60: 262-266, 2001.

10. Zheng X, Johansson M and Karlsson A: Retroviral transduction of cancer cell lines with the gene encoding Drosophila melanogaster multisubstrate deoxyribonucleoside kinase. J Biol Chem 275: 39125-39129, 2000.

11. Zheng X, Johansson $M$ and Karlsson A: Nucleoside analog cytotoxicity and bystander cell killing of cancer cells expressing Drosophila melanogaster deoxyribonucleoside kinase in the nucleus or cytosol. Biochem Biophys Res Commun 289: 229-233, 2001.

12. Roth JA and Cristiano RJ: Gene therapy for cancer: what have we done and where are we going? J Natl Cancer Inst 89: 21-39, 1997.
13. Vile RG, Russell SJ and Lemoine NR: Cancer gene therapy: hard lessons and new courses. Gene Ther 7: 2-8, 2000.

14. Zheng X, Rao XM, Snodgrass C, et al: Adenoviral E1a expression levels affect virus-selective replication in human cancer cells. Cancer Biol Ther 4: 1255-1262, 2005.

15. Sangro B, Mazzolini G, Ruiz J, et al: Phase I trial of intratumoral injection of an adenovirus encoding interleukin- 12 for advanced digestive tumors. J Clin Oncol 22: 1389-1397, 2004.

16. Kirn D, Martuza RL and Zwiebel J: Replication-selective virotherapy for cancer: biological principles, risk management and future directions. Nat Med 7: 781-787, 2001.

17. Zhang Q, Chen G, Peng L, et al: Increased safety with preserved antitumoral efficacy on hepatocellular carcinoma with dualregulated oncolytic adenovirus. Clin Cancer Res 12: 6523-6531, 2006.

18. Liu TC and Kirn D: Gene therapy progress and prospects cancer: oncolytic viruses. Gene Ther 15: 877-884, 2008.

19. Kirn DH and Thorne SH: Targeted and armed oncolytic poxviruses: a novel multi-mechanistic therapeutic class for cancer. Nat Rev Cancer 9: 64-71, 2009.

20. Heise C, Sampson-Johannes A, Williams A, McCormick F, Von Hoff DD and Kirn DH: ONYX-015, an E1B gene-attenuated adenovirus, causes tumor-specific cytolysis and antitumoral efficacy that can be augmented by standard chemotherapeutic agents. Nat Med 3: 639-645, 1997.

21. Reid T, Warren R and Kirn D: Intravascular adenoviral agents in cancer patients: lessons from clinical trials. Cancer Gene Ther 9: 979-986, 2002.

22. Doronin K, Toth K, Kuppuswamy M, Ward P, Tollefson AE and Wold WS: Tumor-specific, replication-competent adenovirus vectors overexpressing the adenovirus death protein. J Virol 74 : $6147-6155,2000$

23. Hermiston T: Gene delivery from replication-selective viruses: arming guided missiles in the war against cancer. J Clin Invest 105: 1169-1172, 2000

24. He XP, Su CQ, Wang XH, et al: E1B-55kD-deleted oncolytic adenovirus armed with canstatin gene yields an enhanced antitumor efficacy on pancreatic cancer. Cancer Lett 285: 89-98, 2009.

25. Arner ES, Spasokoukotskaja T and Eriksson S: Selective assays for thymidine kinase 1 and 2 and deoxycytidine kinase and their activities in extracts from human cells and tissues. Biochem Biophys Res Commun 188: 712-718, 1992.

26. El-Serag HB and Rudolph KL: Hepatocellular carcinoma: epidemiology and molecular carcinogenesis. Gastroenterology 132: 2557-2576, 2007.

27. Hermiston TW and Kuhn I: Armed therapeutic viruses: strategies and challenges to arming oncolytic viruses with therapeutic genes. Cancer Gene Ther 9: 1022-1035, 2002.

28. Wildiers J and De Clercq E: Oral (E)-5-(2-bromovinyl)-2'deoxyuridine treatment of severe herpes zoster in cancer patients. Eur J Cancer Clin Oncol 20: 471-476, 1984.

29. Hui YF and Reitz J: Gemcitabine: a cytidine analogue active against solid tumors. Am J Health Syst Pharm 54: 162-170, 1997.

30. Leitner S, Sweeney K, Oberg D, et al: Oncolytic adenoviral mutants with E1B19K gene deletions enhance gemcitabineinduced apoptosis in pancreatic carcinoma cells and anti-tumor efficacy in vivo. Clin Cancer Res 15: 1730-1740, 2009. 